\title{
LAS CIENCIAS NATURALES Y LOS VALORES
}

\author{
ALBERTO CORDERO \\ Universidad Peruana \\ Cayetano Heredia
}

Una de las características de la epistemología anglosajona de los años 50 y parte de los 60 es la convicción con la cual muchos de sus personajes asumieron y desarrollaron las tesis del positivismo. La doctrina general resultante, llamada "The Received View" (RC) por H. Putnam (Pu 62), era clara, ambiciosa $\mathrm{y}$, sobre todo, sensata a simple vista. Convergían en ella mucho del verificacionismo del primer Ayer (Ay 36) y el refutabilismo del Popper de Po 35. Era una doctrina propensa a abrazar dicotomías radicales: lo analítico vs. lo sintético, teoría vs. observación, contexto de descubrimiento $v s$. contexto de justificación y hecho vs. valor. De ella surgía una visión de la ciencia, entendida como un modo purificado de la razón comprometido únicamente con la verdad. Esta, por su parte, se tomaba como neutral frente a las creencias humanas; la verdad se imaginaba simplemente accesible y carente de toda problemática profunda. En Po 35/59 y Po 65, Popper proclama la vigencia de la teoría correspondentista, explicitada en su opinión por la teoría de Tarski.

RC despachaba, de manera integrada y plausible, varios problemas. No sólo ofrecía una teoría del significado de los términos que refieren a situaciones empíricas no observables ("teóricas") : ofrecía también una doctrina acerca de la justificación de los enunciados enriquecidos por dichos términos. $\mathrm{RC}$ era simple, optimista y conforme con la buena voluntad del sentido común científico. Abandonar el correspondentismo y las dicotomías de las cuales $\mathrm{RC}$ era filial, parecía, desde esta perspectiva, abandonar el rigor lógico a cambio de una vana ilusión de profundidad. 
Sin embargo, el radicalismo de $\mathrm{RC}$ condujo a una reacción. Hacia fines de los años 60 la atmósfera cultural experimentaba, por el lado teórico, la influencia del 2o. Wittgenstein y, por el lado más práctico, el peso de ciertos relativismos propiciados por las ciencias sociales. Pues bien: los cargos contra RC se beneficiaron de esa atmósfera y cuando fueron presentados surgieron tanto desde la propia tradición empirista como a partir de otras tradiciones. Por su parte, Quine $(\mathrm{Qu}$ $53, \mathrm{Qu} 60$ ) había iniciado ya en los años 50 un agudo programa de demolición de los mitos de $\mathrm{RC}$ y de reconsideración crítica del lenguaje científico. De otro lado, empezó a ganar credibilidad la tesis de que la filosofía anglosajona de la ciencia, trivializada por una excesiva búsqueda de claridad y rigor, estaba abandonando la profundidad e integridad propias de la filosofía.

Los ataques contra $\mathrm{RC}$ resultaron beneficiosos de diversas maneras: una saludable aproximación por parte de algunos filósofos anglosajones a los esfuerzos de importantes filósofos europeos (pensadores como Apel o Habermas, para citar apenas dos casos); una reconsideración más justa de ciertas teorías que se presentan a sí mismas como especialmente profundas y que son filosóficamente muy problemáticas respecto de su objetividad, como el marxismo o el psicoanálisis. Muy en especial, sin embargo, el mayor beneficio resultante de los ataques contra RC bien pudiera ser el retorno al seno de la epistemología de un amplio debate sobre los fundamentos de la ciencia y de la epistemología misma. En efecto, como resultado la filosofía de la ciencia, luego de un largo tiempo de "especialización" y dejando de lado muchos mitos y malas costumbres, ha empezado a reincorporarse a los debates más fundamentales de la filosofía general.

Poco queda hoy de las dicotomías analítico/sintético, teoría/observación, descubrimiento/justificación (véanse, por ejemplo $\mathrm{Pu} 75$, Sh 80, Sh 81). En cambio la reconsideración del mito positivista de la dicotomía hecho/valor ha resultado, al parecer, la más difícil. Reconsiderarla es un reto doblemente importante para la filosofía post-kuhniana de la cien- 
cia: 1) por el tema mismo (¿cuáles son los límites de la objetividad?), y 2) porque pone a prueba, en el difícil campo de la teoría de los valores y la objetividad, la tradición empirista. Por lo demás, desde la publicación de $\mathrm{Ku} 62$, este reto ha sido aceptado por varios filósofos; bástenos mencionar a H. Putnam ( Pu 80), M. Hesse (He 74) y Shapere (Sh 81), entre los más preocupados. Quizás uno de los proyectos más ambiciosos en este último sentido sea el esbozado por Putnam en $\mathrm{Pu} 80$, que tomaré como punto de partida del presente ensayo. Las tesis de Putnam son ampliamente discutibles, como espero mostrar; pero eso, en mi opinión, es secundario con respecto a tres importantes aspectos de los planteamientos hechos en $\mathrm{Pu}$ 80:

1) Su intento de demostrar que la filosofía puede abordar fructíferamente el problema de los valores presupuestos por las ciencias naturales.

2) La idea de que tal abordaje puede hacerse dentro de los causes generales de la tradición anglosajona; y

3) El enorme atractivo de ciertas tesis presentadas en $\mathrm{Pu}$ 80 , al menos a nivel de intuiciones (ésto es, argumentos aparte).

\section{La distinción hecho/valor según Putnam}

La primera tarea de este ensayo consiste, por consiguiente, en presentar un resumen de la posición planteada en $\mathrm{Pu} 80$, lo cual hace necesaria una advertencia preliminar. Es conocida la capacidad de Putnam para sugerir ideas atractivas, $\mathrm{Pu} 80$ es en este sentido una contribución particularmente rica, susceptible quizá de varios resúmenes alternativos. Lo que sigue a continuación es simplemente una selección de ideas presentes en $\mathrm{Pu} 80$ y que me parecen importantes para los fines de este ensayo.

$\mathrm{Pu} 80$ contiene en particular tres apetecibles tesis relacionadas íntimamente entre sí.

1) Que la distinción hecho/valor es desesperanzadoramen- 
te borrosa, porque toda categorización fáctica depende de valores.

2) Que no puede haber una dicotomía hecho/valor: todo hecho depende de algún valor y todo valor se materializa en algún hecho.

3) Que lo que tomamos por el mundo real depende de nuestros valores.

La estrategia de Putnam para sacar adelante estas tesis consiste en examinar de cerca el concepto de verdad presupuesto por la presunta dicotomía hecho/valor. Si el fundamento de dicha dicotomía es que sólo los enunciados fácticos - nunca los enunciados valorativos- pueden ser verdaderos, entonces - razona Putnam - una demostración de que la verdad no puede pensarse fuera de un contexto de valores pondría de manifiesto algunos dogmatismos inherentes a la dicotomía hecho/valor. Putnam, en efecto, considera el problema de la verdad desde una perspectiva kantiana post-wittgensteiniana, post-quineana y encuentra que el concepto de verdad no puede ser ni simple ni primitivo. ¿Cuáles son, entonces, los fundamentos de la verdad? Se dice que un cierto enunciado es verdadero no porque éste "corresponde" al mundo en sí (los seres humanos no tenemos acceso directo al mundo en sí), sino porque resulta racionalmente aceptable en un marco coherentista de la verdad, en el cual no hay lugar para enunciados aislados sino para sistemas holistas de ellos. La verdad de un enunciado resulta ser de esta manera una función de nuestros criterios de aceptabilidad racional, los que, según Putnam, a su vez dependen nada menos que de nuestros valores éticos. Esta intromisión de la ética en la verdad científica no es, sin embargo, arbitraria. Dado que no accedemos directamente al mundo y que nuestras teorías son (o forman parte de) sistemas creenciales holistas, los seres humanos estamos en permanente peligro de caer en viciosos círculos epistémicos. Putnam utiliza las atractivas metáforas naturalistas de la enfermedad (para caracterizar los sistemas creenciales fuera de control) y del florecimiento (para los sistemas de mayor 
excelencia) : el ser humano, en opinión de Putnam, florece cognitivamente en ciertos sistemas (por ejemplo las ciencias naturales) y se empobrece y enferma en otros (por ejemplo la astrología, el ocultismo o el super benthamismo). Los primeros son, pues, los sistemas virtuosos que deben merecer nuestra preferencial "racional". Hay aquí, por consiguiente, dos supuestos importantes: 1) que contamos con una sensibilidad filosófica y científica capaz de ayudarnos a identificar tanto los sistemas creenciales en armonía con nuestras capacidades cognoscitivas como aquellos que las deprimen; 2) que los sistemas en armonía con nuestras capacidades cognoscitivas son sistemas virtuosos.

La determinación de los sistemas virtuosos es, para Putnam, una importante tarea empírica: una vez detectados varios sistemas virtuosos y varios enfermizos, debe procederse a extraer, por comparación y contrastación, las notas de la aceptabilidad racional vigente, de hecho, en los sistemas virtuosos escogidos. ¿Cuáles son, entonces, las virtudes responsables del florecimiento cognitivo del ser humano? Putnam distingue cuatro virtudes fundamentales en lo que él llama una buena "imagen subjetiva del mundo": coherencia, amplitud, eficacia instrumental y simplicidad funcional. Así, si la verdad coherentista depende de la fundamentación, ésta, según Putnam, a su vez descansa en criterios sostenidos por los pilares de los cuatro valores anteriores. Pero la verdad es sólo una parte de la aceptación racional: una segunda parte está dada por la relevancia contextual de lo que se dice. Pues no basta que las descripciones sean verdaderas; han de ser también relevantes, ésto es, deben centrarse en un marco conceptual, en algún marco de categorías.

$¿$ Cómo intervienen, en consecuencia, los valores en las ciencias? En primer lugar como presupuestos sociales de nuestro marco conceptual. Ello no quita fuerza a los hechos, pues, en la medida que los valores son presupuestos sociales de categorías descriptivas, los enunciados científicos pueden ser descriptivos y sostenerse en un marco de valores. ¿Cómo es 
eso posible? Putnam es aquí claro y terminante: el acceso a la base conceptual de un enunciado (finalmente a la descripción que éste pueda realizar) no presupone que se comparten los valores sociales sobre los cuales descansa el marco conceptual respectivo. De esta manera, los hechos y la verdad no dependen de preferencias individuales; dependen de preferencias comunales y de ello se sirve Putnam para plantear la tesis más fuerte de $\mathrm{Pu}$ 80: lo que tomamos por el mundo real, dice, depende de nuestros valores, al punto que comunidades con valores diferentes no pueden sino vivir en mundos de tex. tura diferente. La conclusión es, entonces, que nuestro mundo empírico es parcialmente "físico" y parcialmente humano: la mente y el mundo constituyen conjuntamente la mente y el mundo. Y, por cierto, los valores de los que depende la textura del mundo son valores éticos. Putnam compara su versión del problema de la distinción hecho/valor con distinciones más populares basadas en presunciones de consenso respecto de los hechos: presunciones al estilo del sentido común, de convergencia frente a los hechos (al estilo del llamado "método científico") y de reducción fisicalista del dominio fáctico (al estilo de Quine). Todas estas distinciones alternativas son, afirma Putnam, infructuosas: los hechos dependen siempre de algún valor y todo valor materializa algún hecho. $\mathrm{Y}$, por consiguiente, debe esperarse que cierta comunión de valores por parte de un grupo humano traiga consigo una correspondiente comunión de opiniones; en definitiva, el advenimiento de un mundo humano común. ¿Es, por consiguiente, pensable una comunión humana plena? Putnam reconoce liberalmente que no.

Hay muchas otras ideas sugerentes en $\mathrm{Pu} 80$, particularmente acerca de la ética y la epistemología de la ética (temas de suyo centrales en $\mathrm{Pu} 80$ ). Me parece, sin embargo, que lo dicho resume los elementos de $\mathrm{Pu} 80$ relevantes al problema de la distinción ciencia natural/valor, los cuales, en esa medida, tomo aquí como punto de partida. 


\section{Algunos problemas}

Los planteamientos anteriores, de suyo tan atractivos, son 一qué otra cosa podían ser-problemáticos.

En primer lugar está la tesis de que la distinción hecho/ valor es brumosa al punto de afirmarse que los hechos y los valores se sostienen mutuamente a través de un delicado juego. Es una tesis muy plausible desde el punto de vista crítico, pero que no parece estar a la altura de las sugerencias dejadas alrededor de ella en Pu 80. ¿Es razonable pensar con Putnam que lo que tomamos por el mundo real depende de nuestros valores? Depende, naturalmente, de los alcances que queramos dar a las palabras. En particular, depende de la posición que se tome frente a ciertas preguntas que $\mathrm{Pu} 80$ pareciera dejar a sus lectores, especialmente las siguientes:

1) Si lo que tomamos por el mundo depende de nuestros valores, ¿de qué manera específica varía la textura del mundo con esos valores?

2) Si la verdad depende de las virtudes epistémicas de coherencia, eficacia instrumental, amplitud y simplicidad funcional, es claro que el dominio de esta dependencia habrá de ser cierto compromiso entre las cuatro virtudes, nunca una sola de ellas con exclusión de las otras. Pues, bien: ¿qué compromisos específicos entre dichas virtudes apuntalan a la verdad y cómo lo hacen?

3) Aún asumiendo que los hechos dependen de los valores, a primera vista una cosa es la objetividad de las ciencias naturales y otra muy distinta la objetividad de, por ejemplo, el arte, o incluso las ciencias sociales. ¿Qué es lo que distingue, pues, a las ciencias naturales (física, química y biología, para precisar mejor la cuestión) de otras ciencias o de otras empresas cognoscitivas?

Las anteriores son, convicta y confesamente, cuestiones de detalle; en mi opinión detalles esenciales, sin embargo, para 
sacar adelante las tesis que Putnam tiene en mente. Por cierto, el problema $-\mathrm{y}$ me atrevería a decir que también la fuerza- de la posición planteada en $\mathrm{Pu} 80$ reside en su carácter fundamentalmente programático. $\mathrm{Pu} 80$ es ante todo un aperitivo filosófico. Su fuerza reside, por un lado, en el programa de investigación que plantea y que en gran medida cada lector suyo puede articular según sus intereses (en el presente ensayo, en términos de las preguntas 1-3). Pero hay al menos un segundo lado, quizá hasta más importante que el primero y es el relacionado con nuestra capacidad científica y filosófica para identificar tanto empresas humanas "fructíferas" como empresas "enfermizas". Un segundo lado que plantea una $4^{\mathrm{a}}$ pregunta más bien monumental:

4) ¿No tenemos acaso la capacidad de detectar la superioridad humana de, digamos, la física de Newton frente a la "obvia" perversión de la astrología contemporánea? La pregunta, por desgracia, habla por sí sola y si bien a este respecto $\mathrm{Pu} 80$ sugiere mucho, un grave problema es que calla aún más.

En lo que sigue trataré de abordar algunos aspectos centrales de las preguntas 1-3. No dejo de lado la 4 a pregunta porque crea que carece de importancia (todo lo contrario); ocurre simplemente que no tengo mucho que decir al respecto, como no sea que comparto la atracción de una respuesta naturalista para ella —que, debe sospecharse, habrá de ser vastamente compleja.

\section{Algunos problemas de vocabulario}

Putnam, como muchos autores, deja implícitos algunos términos de las proporciones filosóficas de "valor" y "virtud". Aunque, en general, el tema de la ciencia y su relación con los valores se ocupa de problemas acerca del valor, la virtud y la verdad, una cuestión presupuesta irremediablemente por esos problemas es cómo, por qué y con qué consecuencias de- 
seamos conocer. Conviene, por lo tanto, intentar algunas precisiones en relación con aquellos términos antes de seguir adelante con las preguntas planteadas en la sección anterior. Sólo algunas discutibles precisiones, no soluciones. Y únicamente para facilitar la lectura de lo que sigue. No pretendo, quede esto claro, interpretar aquí a ningún autor en particular.

Entenderé, discutiblemente, que los valores remiten a nuestros deseos. Así, en particular, el deseo de conocer hace valioso el conocimiento y virtuoso todo aquello que perfecciona las actividades públicas destinadas a procurarlo. Podemos hablar, por consiguiente, de virtudes epistémicas como aquellas virtudes que expresan valores fundamentales en la disposición humana para conocer. Conocer, tratar de comprometerse sólo con aquellas creencias que, estando debidamente fundamentadas, son además verdaderas, es ante todo una manera de sobrellevar la inmarcesible riqueza de nuestro mundo empírico. Mediante el conocimiento se logra, quizá, cierta serenidad lúcida frente al mundo y la vida. Debajo, yace siempre, sin embargo, un brumoso e inextricable juego de disposiciones humanas. Son las disposiciones que encuentran su expresión natural en la actividad cognoscitiva, nuestras disposiciones "epistémicas".

Como para cada una de las actividades humanas, para el conocimiento son pensables algunos defectos y algunas virtudes. Las virtudes epistémicas son, por consiguiente, ciertos elementos que, completando nuestras disposiciones cognoscitivas, las articulan - vale decir, las perfeccionan- de suerte que cumplan un máximo de los objetivos planteados por ellas. La coherencia, la eficacia instrumental, la simplicidad funcional y la extensión de dominio empírico de un sistema creencial poseen, de esta manera, cada uno un rostro de valor y otro de virtud. Constituyen valores, porque convienen a nuestras disposiciones epistémicas. Conducen a la virtud en la medida que orientan nuestra actividad cognoscitiva hacia la realización de esas disposiciones.

Por su parte, el conocimiento requiere de la verdad. El conocimiento apunta hacia la verdad; pero, ya lo sabemos, "la 
verdad es el lugar común de demasiadas cuestiones filosóficas profundas". En lo que concierne a este ensayo, el problema de la verdad se proyecta de dos maneras relacionadas entre sí, pero de ninguna manera idénticas. Por un lado está la cuestión del significado de la palabra "verdadero"; por el otro, la cuestión acerca de los criterios que nos permiten decidir si un enunciado es verdadero o falso.

Ambos aspectos del problema de la verdad son, naturalmente, demasiado complejos y ambos exceden alevosamente los fines de este trabajo. Las posiciones frente a ellos son muchas, pero una que goza de la especial simpatía de quien esto escribe es aquella que, dentro de un marco general coherentista, intenta salvar el máximo de intuiciones correspondentistas. No es ésta la ocasión apropiada para emprender su defensa, pero el punto es más o menos el siguiente: 1) si el falible entendimiento humano está basado en creencias imposibles de confirmar de modo absoluto, entonces debemos admitir que nuestras creencias tienen el carácter de interpretaciones del mundo y de la vida. 2) Las ideas, especialmente las tesis científicas, están llamadas a realizar las disposiciones epistémicas mencionadas al principio de esta sección. 3) Todo sistema de creencias nace, por consiguiente, hipotecado a un fin activo y contemplativo que debe satisfacerse. 4) Un fin siempre parcial, selectivo, respecto del cual nuestras creencias deben ser útiles sin dejar por ello de ser sinceras o no. bles. Aún en el caso de la ciencia natural puede decirse que ellas no son ni sólo ni únicamente fieles al mundo empírico. La fidelidad absoluta, imparcial, es sencillamente imposible.

Así pues, si la ciencia y el conocimiento humano son finitos y parciales, la estrategia óptima bien pudiera ser la de insistir, volviendo una vez más la mirada hacia C. S. Peirce, en la construcción de alguna forma de pragmatismo semántico (totalmente alejado, ésto es, de cualquier pragmatismo referente a la verdad).

Realidad y valoración

Hechas las anteriores precisiones y observaciones, pasemos 
a considerar la primera pregunta que nos hemos planteado en este ensayo, esto es, el papel que juegan los valores en la textura específica de lo que tomamos por mundo real.

Una idea fértil en este campo pareciera ser la de subdeterminación empírica de las teorías y el lenguaje público en general. La idea es relativamente sencilla y presenta dos aspectos fundamentales: 1) que nuestro mundo empírico es tan rico en elementos discernibles que es imposible reducirlo plenamente a un lenguaje; y 2) que nuestras sistematizaciones, categorizaciones y teorías pueden "corresponder" al mundo, nunca ser determinadas unívocamente por el mundo empírico. Vivimos, en este sentido, en un mundo inefable, guiados por teorias sobre las cuales penden problemas tan profundos como los de la inducción y la categorización lingüística; un mundo naturalmente inconexo, articulado con cierta arbitrariedad por nosotros. Se parte aquí, por cierto, de la tesis de que las "arbitrariedades" empíricas de los lenguajes naturales y las de las teorías empíricas, lejos de ser triviales, proceden de una falta natural en nuestro mundo empírico: su falta de articulación.* Por consiguiente, son primeramente nuestras preferencias comunales las responsables de los lineamientos abstraídos a partir de la indefinidamente rica información potencial contenida en nuestro mundo. Vale decir, ciertos aspectos abstraídos a expensas de muchos otros no obstante discernibles.

Consideramos el problema. Parecería a simple vista sensato pensar que nuestro mundo empírico admite una suerte de clasificación "natural", ésto es, bien una clasificación inicial de suyo estable o bien una clasificación en la cual los seres humanos "razonables" estamos destinados a converger. Muchas consideraciones, por desgracia, indican que ese no es

* Un ejemplo de "cierta arbitrariedad" en la articulación de un dominio enpírico dado es el correspondiente a, por poner el caso, una habitación. Esta puede pensarse de un número indefinidamente grande de maneras distintas (si bien no necesariamente incompatibles): su propio "espacio", para empezar, puede concebirse en términos visuales o auditivos; y luego uno puede concentrar la atención en diferentes aspectos: los subespacios de la habitación, las cosas, las propiedades "continuas", y así sucesivamente. 
el caso: razones psicológicas, cibernéticas, histórico-científicas y de la teoría de la confirmación empírica. Razones que nos hacen sospechar de la presencia de valores en el seno de los lenguajes ordinarios y de la ciencia. Unidas a las razones generales derivables del problema de la inducción, las razones científicas constituyen un marco propicio a las sospechas en cuestión.

Ahora bien, la pregunta es ¿por qué habríamos de encontrar valoraciones en nuestras ciencias naturales? Pensemos, para empezar, en una clasificación "dada" y medianamente rica, de cualquier dominio empírico (pensemos, para hacer el ejercicio más concreto, en el clominio de los quehaceres llanamente cotidianos). El más mínimo deseo de utilizar esa clasificación para orientarnos dentro del dominio que le es propio haría indispensable cierta sistematización conceptual: identificaciones, reidentificaciones, la clasificación tendería a complementarse con generalizaciones y uniformidades "empíricas". ¿Empíricas? En lo fundamental debiera admitirse que sí; sin olvidar, por cierto, el fin pragmático, orientador, que motiva su búsqueda en primer lugar. La complejidad y riqueza de aspectos y matices presentes en el mundo empíri. co no va, como ya nos ha acostumbrado a sospechar Hume, de la mano con nuestra necesidad de sistematizarlo. Hay, pues, un serio conflicto entre, por un lado, la complejidad y, por otro, la utilidad de una clasificación (la utilidad se toma aquí en el sentido semántico especificado anteriormente en relación con los problemas de la verdad). Se trata, en último término, de un viejo conflicto preliminar de intereses: la riqueza descriptiva contra la utilidad de las descripciones.

Como decía, la naturaleza de este conflicto no ha pasado desapercibida en disciplinas como la cibernética, la lingüística chomskiana, la teoría de la inferencia y la confirmación inductivas, la psicología de la percepción y la psicología genética. En efecto, todas estas disciplinas han dado lugar a sospechas convergentes respecto del conflicto riqueza-descriptiva/ventaja-descriptiva. Todas estas ciencias convergen en la idea de que, en primer lugar, la racionalidad y la sensibilidad 
humanas requieren de moldes sistematizadores para entrar en resonancia cognitiva con el mundo empírico. Pero eso no es todo; ellas convergen también en la sospecha complementaria de que no bastaría que tales moldes fueran únicamente formales, digamos únicamente lógicos: se necesitan moldes materiales. Se necesitan preconcepciones del mundo empírico: un molde espacial, un molde temporal, otro causal (la mecánica cuántica parece sugerir incluso la presencia de un molde lógico-empírico). Y, más gravemente, se necesitan preconcepciones de que el mundo es de "cierta" manera, unidas al sentimiento de que tal manera es o no deseable, ésto es, se necesitan apreciaciones valorativas. El mundo empírico es simplemente demasiado rico para nosotros. Si acaso podemos contemplarlo cabalmente (algo muy dudoso), de ninguna manera podemos articularlo totalmente. Para articular hay que empobrecer. Y en este sentido articular nuestro mundo es resignarse a perder información potencial acerca de él. Encontramos, por lo tanto, al menos dos centros de valoración fácilmente discernibles: un centro epistémico y otro de relevancias (instintivas, culturales o individuales).

No se afirma en lo dicho nada especialmente profundo. Las apreciaciones valorativas han ocurrido y ocurren por todas partes. Por ejemplo, que la materia es de dos tipos: terrenal y celestial, y que la materia celestial debe conformar todas nuestras normas de perfección; que parte del mundo es obra de los dioses del bien y parte de los dioses del mal, y que hay que dominar la parte que procede del mal; que hay seres humanos y hay un mundo natural, y que repugna a la dignidad liumana todo intento de reducir la mente del hombre al mun. do físico... Pues bien: el impacto de valoraciones de este tipo es inmediato; ellas imponen un marco no natural de relevancias diferenciales a todos nuestros dominios empíricos. Marcos importantes, porque orientan nuestra sofisticación descriptiva aplicada al mundo empírico y contribuyen, de esta manera, a "darle" textura.

¿Qué debemos, pues, esperar de los presuntos moldes sis- 
tematizadores de la mente humana? Aparte de la presencia de una sólida componente lógico-formal, de lo dicho se desprende que la mente (como la inteligencia artificial propiamente dicha) parece requerir de marcos conceptuales, marcos sintéticos, en el lenguaje de Kant, y marcos valorativos, ambos relativamente a priori. Hay en ésto, sin duda, dos referencias sospechosas: una a la inteligencia artificial; otra a ciertos marcos "relativamente a priori". La primera es sospechosa, o no, según la idea que se tenga de la naturaleza de la inteligencia. Qué cosa es la inteligencia, aparte de un título de cortesía que damos a determinadas disposiciones y capacidades, es una cuestión que depende de los valores que tengamos en la mente al hablar de inteligencia humana; vale decir, justo nuestro tema. Del otro lado, la idea de que la mente necesita ciertos moldes relativamente apriorísticos no es, en mi opinión, ni extravagante ni reaccionaria. Se habla de moldes óptima pero no absolutamente insensibles a la experiencia. Se habla de moldes que pueden cambiar y que, de hecho, hemos cambiado muchas veces desde la antiguedad más remota (las ciencias naturales últimamente nos han enseñado a cambiarlos con más facilidad que de costumbre). Son moldes que debemos pensar como disposiciones de nuestra mente, no como estructuras "necesarias". Los moldes sistematizadores resultarían ser, en consecuencia, preconcepciones relativamente a priori; moldes parcialmente condicionados por nuestra biología (lógica común, geometría euclideana, intuiciones micro-inductivas, distinción ente/atributo...) y parcialmente condicionados por nuestra cultura (continuidad del espacio-tiempo, supremacía de la persona). Se trata de estructuras preliminares, pero no por ello necesariamente nocivas (útiles, por ejemplo, para facilitar nuestra adaptación al mundo físico y al mundo humano "inmediatos").

Hay, por lo tanto, un sentido en el cual puede aceptarse que "la textura de lo que tomamos por el mundo real depende de nuestros valores". Pero los valores aludidos en ese sentido son de carácter comunal; no sirven de presupuesto a empresas individuales, sino al lenguaje ordinario y a las ciencias natu- 
rales, ambos de carácter proverbialmente públicos. Un cambio en los valores comunales de un grupo irá, según lo dicho, acompañado de un cambio del marco público de relevancias, lo cual a su vez conducirá a que dicha comunidad reoriente la sofisticación descriptiva de su lenguaje y de su ciencia en una nueva dirección. Con los nuevos valores, pasan al olvido muchas notas del mundo empírico tenidas anteriormente por relevantes. Son sustituidas por aspectos hasta entonces venidos a menos o incluso desatendidos (un caso típico es el de la revolución científica de los siglos XVI-XvII).

$¿$ Añaden algo los valores? Hemos observado que añaden textura. Pero esa particular textura del mundo es una función de nuestros valores, no porque éstos añadan real y positivamente algo al mundo, sino porque, por el contrario, nuestros valores nos ayudan a que dejemos de lado (en algún sentido, despreciemos) un rico e indefinido campo empírico. No necesariamente para siempre, por cierto. Lo que se pierde se conserva en el mundo como una suerte de información potencial a disposición del resto del tiempo; a disposición de la ciencia, el arte y la filosofía, quizá también la religión, del futuro.

\section{Verdad y virtud}

Las dos cuestiones siguientes en nuestra lista son: a) qué compromisos entre las virtudes epistémicas identificadas anteriormente constituyen la base de la verdad en las ciencias naturales; y b) cómo hemos de entender la distinción entre las ciencias naturales y otros modos de conocimiento. Creo que la primera cuestión impone una tarea inevitable si queremos sacar provecho de la tesis de que la verdad depende de valores. En efecto, decir que la verdad depende de valores tales como la coherencia, la eficacia instrumental, la extensión y la simplicidad funcional es intuir mucho, pero sin casi decir realmente nada de provecho. Todo discurso es, "a su manera", coherente, instrumentalmente eficaz (vale decir, útil), 
etc. Se trata, por lo tanto, de especificar menos ambiguamente cuáles son estas virtudes en el caso de las ciencias naturales.

La segunda pregunta de esta sección, se limita a solicitar algo razonable: que se aclare la innegable diferencia epistemológica que separa cognitivamente a la física, la química y —cada vez más — la biología de, por ejemplo, el psicoanálisis, el marxismo, el arte o la filosofía. Que esto se aclare, por supuesto, admitiendo la presencia de valores en el seno de las ciencias naturales - no a la manera positivista - y que se aclare sin presuponer necesariamente un mayor valor cognoscitivo favorable a las ciencias naturales. Hay que averiguar, por lo menos, si se trata de una diferencia solamente de grado o de una diferencia mayor, una diferencia de tipo -y si fuera lo último, de qué tipo.

¿Cómo, sin embargo, ha de tratarse la primera cuestión? Realistamente, debiéramos esperar que los presupuestos valorativos de las ciencias naturales fuesen tan variados como los grupos sociales de investigadores que las cultivan. No es, después de todo, ningún secreto el comportamiento algo tribal de muchísimos científicos. Así pues, ¿cómo hemos de proceder en este caso? Está claro que se desea fortalecer, con un mínimo de prejuicios, algunos valores fundamentales de amplia relevancia epistémica: los valores de los que depende del modo más inmediato la verdad científico-natural.

Me parece que un método apropiado para tratar el caso que nos ocupa pudiera ser el siguiente: preguntémonos cuál es la imagen de la ciencia más popular y recurrente entre los físicos (asume aquí que los químicos y la creciente población de biólogos fisicalistas aceptan la preeminancia de la física sobre sus respectivas disciplinas). Dejemos de lado si dicha imagen es o no razonable; no nos preocupemos de lo epistemológicamente caricaturesca o absurda que ésta puede ser. Los físicos, en general, no son filósofos profesionales (ni falta que les hace para cumplir sus objetivos usuales). No son filósofos, pero demuestra ser propensos a considerarse objetivos, los más verdaderos y honestos; en fin, propensos a considerarse el ideal de éxito epistémico que, dicho sea de paso, son en buena parte. 
Y demuestran también ser muy sensibles contra la posibilidad de que se confundan sus méritos con los en opinión de la mayoría de ellos - más dudosos éxitos de las ciencias sociales, el arte y — qué duda cabe - la filosofía. La pregunta es, por consiguiente, ¿ $\mathrm{Ha}$ producido la física contemporánea una visión epistemológica, un mito predilecto acerca de sí misma? Y de ser así, ¿cuál es ese mito?

Varios grandes nombres vienen a la mente: Einstein, el círculo de Viena, Bohr, Frank, Bridgman, Eddington, Hanson... Varios nombres y también varios grupos: fenomenalistas, dispuestos a creer que la ciencia tiene por función primordial una económica sistematización predictiva de los datos sensoriales; operacionalistas, convencidos de la privilegiada posición de las sistematizaciones numérico-predictivas de las operaciones experimentales; positivistas capaces de sugerir que la explicación y la predicción científicas son equivalentes; y atisbos intermitentes de realismo de corte Popperiano. Todas éstas son, sin duda, caricaturas, pero caricaturas todas muy seriamente dedicadas a resaltar el valor metodológico de la predicción en las ciencias; bien en beneficio de la verificación, bien en beneficio de la refutación, de la explicación fundamentada o del control predictivo del medio ambiente. Y de caricaturas nada arbitrarias: es un hecho que muchos físicos profesan las más bastas tesis instrumentalistas.

¿Cuáles son, pues, según todas esas visiones, las virtudes cognitivas que aseguran para las ciencias naturales un estatuto epistémico especial?

Destaca en primer plano el ideal de control predictivo. En segundo plano, pero también de manera fundamental, están la economía de entidades teóricas, la coherencia, la simplicidad descriptiva y la extensión. Pero se observa una marcada preferencia en favor de la predicción. Y se observa también que al menos dos virtudes epistémicas generales admiten versiones bastante específicas en la física:

i) La eficacia instrumental, entendida como control predictivo; $\mathrm{y}$ 
ii) La simplicidad funcional, bifurcada en por lo menos dos aspectos:

a) economía de entidades teóricas; y

b) simplicidad notacional-descriptiva.

Ahora bien, si esta visión de los valores internos de las cien. cias naturales fuera correcta, resultaría que no todos los valores identificados se encuentran en igual pie respecto de sus efectos sobre la verdad. Por el contrario, habría una jerarquía cuya estructura podemos resumir en 3 principios:

(1) El control predictivo es una virtud fundamental de las ciencias naturales.

(2) La coherencia, la extensión de dominio empírico, la economía de entidades teóricas y la simplicidad notacional son virtudes fundamentales de las ciencias naturales.

Finalmente, pero de manera importante:

(3) El principio (1) es dominante con respecto al principio (2). Esto es, las virtudes mencionadas en el principio (2) deben supeditarse a una máxima realización del valor de control predictivo en las ciencias naturales.

Las evidencias de que el principio (3) concuerda con la práctica científica son muchas. La llamada lógica cuántica, por ejemplo, pretende sacrificar nada menos que la lógica para salvar la causalidad clásica (y con ella el principio más fuerte posible de predicción); la física nuclear o la bioquímica sacrifican constantemente los valores de simplicidad en beneficio del valor de control predictivo; la extensión, ésto es, la más amplia cobertura del dominio empírico, es sacrificada en la psicología conductista en beneficio del control predictivo del comportamiento. No afirmo con esto que las ciencias naturales tienen por objeto el control predictivo de sus respectivos dominios empíricos; por el contrario, su objeto es alcanzar el entendimiento. Metodológicamente, sin embargo, 
están ligadas al control predictivo. La asociación instrumental y pragmática de las ciencias es, por consiguiente, de carácter semántico: la verdad asociada con la fundamentación y ésta con el control predictivo.

De esta manera, si las virtudes epistémicas son las que acabo de especificar; y si como se ha sostenido, la verdad, lejos de ser un concepto primitivo, debe pensarse en función de dichas virtudes, entonces cabe esperar que en las ciencias naturales la verdad de los enunciados esté dominada por ciertos elementos de fundamentación causal. Debiera ser el caso, en particular, que respecto de su confirmación los enunciados de las ciencias naturales tienden a relacionarse entre sí en vastas tramas causales. Podemos, pues, poner a prueba la visión que sobre los valores científicos y el método de identificación de éstos se propone en el presente ensayo.

La destacada función de la causalidad en las teorías físicas no está en discusión; salta a la vista. Ya no se habla científicamente de determinismo, pero tampoco es que se rechace la causalidad. Inmersa en consideraciones estadísticas, algo modificada en algunos dominios empíricos, la causalidad sigue dominando la descripción temporal en las ciencias naturales. La vigencia de la causalidad es, por consiguiente, clara. La cuestión es ver cómo sirve la causalidad a la relación entre la verdad y la justificación en las ciencias naturales.

¿En qué pueden basarse los científicos para asegurarnos que un cierto enunciado es verdadero o falso? La epistemología deja aquí por lo menos dos dimensiones abiertas: 1) la proyección holista del enunciado y 2) los procesos de identificación y reidentificación. La componente holista regula la optimización del patrón de valores epistémicos (coherencia, eficacia instrumental, simplicidad funcional y extensión) en el sistema teórico. No tiene que hablarse aquí, dicho sea de paso, de holismos monumentales. Se viene haciendo cada vez. más claro que el holismo de las ciencias naturales es de alcance relativamente modesto, menos ambicioso que el holismo a la manera de Quine (véase Mo 81). En cualquiera de sus formas, sin embargo, un holismo es tal en la medida que glo- 
balmente orienta la asignación de valores de verdad o falsedad a los enunciados que participan de sus méritos. La verdad en las ciencias naturales es, a la luz de la reciente investigación epistemológica, una propiedad contextual de los enunciados. Esto es, los enunciados son verdaderos o falsos únicamente en un contexto teórico: la verdad en las ciencias naturales se fundamenta formal y materialmente dentro de los alcances de un contexto teórico dado, nunca aisladamente.

¿Favorece el holismo (tal como éste se da en las ciencias naturales) el valor de predicción? La práctica real de la refutación en las ciencias naturales parece responder que sí. Las teorías se suceden unas a otras, pero nunca sin transmitir y perfeccionar su creciente depósito de resultados pragmáticos. No me es posible ni conveniente detenerme aquí a elaborar estas tesis, pero desearía dejar al menos un punto en claro: las leyes naturales, en la medida que son elementos instrumentales, útiles para el control predictivo, no desaparecen luego de una refutación, sino que simplemente son reformulados a nivel global. ¿No es ese, en efecto, el caso incluso bajo las más dramáticas revoluciones científicas? ¿No dejó acaso la teoría de la relatividad restringida intacto el depósito instrumental de la mecánica clásica?

Las leyes usadas localmente (las leyes nunca se usan de manera estrictamente global) a la hora de construir casas, coches o relojes de péndulo siguieron en uso (más aún en uso mejorado). Pues la nueva teoría, aparte de reinterpretar esas leyes, enseña que debemos esperar resultados antes insospechados a velocidades relativas próximas a las de la luz.

Vemos ahora lo que ocurre con la segunda dimensión epistemológica de la verdad. La identificación y la reidentificación de situaciones empíricas (en suma la categorización lingüística de nuestro mundo empírico) es de hecho particularmente pragmática en las ciencias naturales. Qué duda cabe, éstas persiguen la precisión centrada —en la medida de lo posible- en el establecimiento empírico de condiciones necesarias y suficientes para la aplicación de sus términos: " $\mathrm{X}$ es $\mathrm{P}$ si y sólo si $\mathrm{X}$ es A, B y C". Persiguen una precisión 
que nunca logran de modo perfecto; entre otras razones porque todos los conceptos importantes de las ciencias son conceptos arracimados. Pero queda claro que en las ciencias na. turales hay un ideal regulador en torno a la precisión y, más aún, se trata de un ideal regulador causalista: el ideal de que los criterios de identificación y reidentificación empíricalingüística se agoten en la trama nomológico-causal de las ciencias naturales. Desde este punto de vista, las teorías más "serias", más "logradas" son aquellas que mejor satisfacen este ideal regulador. Y, por cierto, ¿no es ese acaso el mayor problema que afrontan institucionalmente ciencias como la biología neodarwinista o la psicología no conductista?

El ideal de someter el lenguaje de las ciencias a una trama nomológico-causal ofrece grandes beneficios. En particular, ofrece un alto nivel de confirmabilidad en lo que respecta a los enunciados básicamente descriptivos: ofrece "hechos". Hechos, entendidos como los referentes de descripciones verdaderas a juzgar por su contexto predictivo.

Carnap hablaba de los predicados observables de manera que, afirmaba

$A$ predicate ' $P$ ' of a language $L$ is called observable for an organism (e.g., a person) $N$, if, for suitable arguments, e.g., ' $b$ ', $N$ is able under suitable circumstances to come to $a$ decision with the help of a few observations about a full sentence, say ' $P(b)$ ', i.e. to a confirmation of either ' $P(b)$ ' or ' $-P(b)$ ' of such a high degree that he will either acept or reject ' $P(b)$ '. (Ca 36/53), p. 63.

La concepción de los hechos ideales de las ciencias naturales a la cual nos han conducido las argumentaciones de este ensayo se encuentra, pues, en cierta consonancia con la teoría carnapiana de los observables: los hechos considerados como los referentes de creencias verdaderas altamente confirmadas.

Pero volvamos ahora al tema central, el problema de la verdad. Si los argumentos presentados hasta aquí son correctos, la verdad no es un término primitivo en la teoría de las 
ciencias naturales, sino un término relacionado con ciertas virtudes epistémicas, articuladas en un patrón dominado por cierta virtud pragmática: la capacidad para el control predictivo. Pero notemos que el control predictivo es un valor optativo. Y también un valor empíricamente limitado, en el sentido de que simplemente no es en absoluto obvio que exista una trama causal capaz de cubrir todas nuestras expectativas teóricas. El reino de los hechos naturales (aquellos cuya verdad está dominada por la virtud pragmática) es un reino limitado por la complejidad y la inestabilidad de ciertos dominios empíricos; por la capacidad de esos dominios de reaccionar ante nuestros intentos de observarlos, de categorizarlos; por su propia indeterminación causal; y —qué duda cabe - por nuestra propia moralidad. En efecto, no sólo ocurre que no podemos - por razones de imposibilidad meramente práctica - constituir, por ejemplo, una teoría antropológica que sea satisfactoria respecto de la virtud de predicción: lo cierto es que a muchos nos repugna incluso la idea de intentarlo. En especial la idea, necesariamente previa, de experimentar, explorar y, a fin de cuentas, especificar con rigurosidad y frialdad "científicas" las posibles relaciones causales que afectan a los seres humanos.

Hechos naturales vs. hechos en general

Finalmente unas palabras sobre los límites del control predictivo y su relación con las variedades de objetividad. Si bien los hechos avalados por el control predictivo son los hechos "óptimos" de las ciencias naturales, debe quedar claro, sin embargo, que no todos los hechos son óptimos en las ciencias naturales. Los hechos pragmaticamente reducidos a su estructura causal son altamente confirmables. Sin embargo, los dominios a los cuales éstos pertenecen exceden, en cualquier momento dado, las estructuras causales cientificamente determinadas en ellos; pues nuestras expectativas empíricas y teóricas simplemente exceden siempre el marco causal disponible. En física o biología teóricas, por ejemplo, se dan 
innumerables casos de dominios empíricos actualmente incontrolables desde el punto de vista pragmático. Dominios abiertos, de esta manera, a la especulación, no reducibles a formas desarrolladas del control predictivo, al menos por el momento. Es el caso, por ejemplo, de los fundamentos de la mecánica cuántica, centro importante de interpretaciones físicas e ideológicas en conflicto.

La conclusión se sugiere a sí misma: la imposibilidad de articular el valor de control predictivo en un dominio científico dado, va acompañada de la revaloración de otras consideraciones menos pragmáticas. Un retorno a veces intenso, como en la mecánica cuántica (recuérdese la disputa Einstein-Bohr y sus ramificaciones). Reprimidos en condiciones normales por el hegemónico valor de control predictivo, los valores e intereses no pragmáticos retornan a la escena de la aceptabilidad racional cuando aquél deja de funcionar.

El control predictivo es, de esta manera, una virtud de alcance limitado. Una pregunta pendiente, por lo tanto, es la de si cabe acaso pensar en alguna forma del valor de eficacia instrumental que no sea el control predictivo.

Entre algunas de las respuestas más radicales dadas a esta pregunta se encuentran las de quienes piensan que son formas válidas de la eficacia instrumental la persuasión revolucionaria o la emancipación política. Delante de quienes opinan de esa manera, sin embargo, yace la monumental tarea de mostrarnos cómo distinguir la simple manipulación mental de los "conocimientos" que pudieran surgir de aquellas formas supuestamente alternativas de la eficacia.

No obstante, la cuestión de las alternativas de la eficacia instrumental -en general, de las virtudes epistémicas- y su relación con el conocimiento humano es importante. Las ciencias naturales se benefician de la hegemonía del control predictivo. Son ciencias que, a no dudarlo, conducen a considerables depósitos de conocimiento. Pero, por otro lado, es un hecho que hay también conocimientos cuya verdad no puede ni dudarse demasiado ni reducirse a meros valores predictivos: nuestro conocimiento de la voluntad y el pensamiento de 
otras personas; el conocimiento que tenemos de otras culturas; el conocimiento que tenemos cuando, a veces, comprendemos a ciertas personas o grupos humanos mejor que ellos mismos. Por no mencionar las conversaciones que muy probablemente todos tengamos en el futuro con robots...; ni mencionar tampoco la objetividad de algunas de nuestras valoraciones.

Tiene que haber lugar, por consiguiente, no sólo para una objetividad-predictiva, altamente cultivada por las ciencias naturales. Hay también otras formas de la objetividad, basadas quizá en otras virtudes no son las estudiadas en este ensayo. Tal vez apenas en las mismas virtudes epistémicas que hacen de las ciencias naturales lo que son, pero sin la hegemonía del control predictivo. $O$ tal vez, de éstas y también de otras virtudes, incluida, por ejemplo, la virtud de buscar la liberación del ser humano. Ciertamente sería importante averiguarlo.

\section{REFERENCIAS}

Ay 36 A. J. Ayer, Language, Truth and Logic. Gollanez, 1936.

Ca 36/53 R. Carnap, "Testability and Meaning" Philosophy of Science 3, (1936) 420; reimpreso en Feigl and Brodbeck, (ed.) Readings in the Philosophy of Science, Appleton-Century Grofts, New York, 1953.

$\mathrm{He} 74$

$\mathrm{He} 78$

M. B. Hesse, The Structure of Scientific Inference. Macmillan, 1974. "Habermas' Consensus Theory of Truth"; en PSA Conference Proceedings, San Francisco, 1978.

Ku 62 T. S. Kuhn, The Structure of Scientific Revolutions. Chicago UP, 1962.

Mo 81 L. U. Moulines, "The Ways of Holism", Actas del II Simposio Internacional de Filosofía, IIF-UNAM, México, 1981. [en prensa].

Po 35/59 K. Popper, The Logic of Scientific Discovery. Hutchinson, 1959.

Po 65

$\mathrm{Pu} 62$

$\mathrm{Pu} 75$

Pu 80

Qu 53

Ou 60

Sh 80 Conjectures and Refutations. Basic Books, 1965.

H. Putnam, "What Theories Are Not"; en Pu 75. Collected Papers, 2 Vols. Cambridge UP, 1975.

"Fact and Value"; conferencia ofrecida en la UPCH, Lima; cap. 6 de Reason, Truth and History. Cambridge UP, 1982.

W. V. O. Quine, From a Logical Point of View. Harvard UP', 1953/62. Word and Object. MIT, 1960.

D. Shapere, "Reference, Necessity, and the Role of Reasoning in Scientific Change"; Actas del I Simposio Internacional de Filosofía. IIF, UNÁM, México, 1981. [en prensa].

Sh 81 "The Rolt of Observation in Science"; Actas del II Sim. posio Intesnacional de Filosofía, IIF, UNAM, México, 1982. [en prensa]. 
SUMMARY

Cordero reconsiders - from the post-Kuhnian view of Natural Science - the fact/value dicotomy. His analysis involves: (1) inquiring about the limits of objectivity, and (2) challenging the empiricist tradition in the areas of value theory and objectivity. The author examines Putnam's position, according the which, a fact always depend upon a value and every value materializes itself in a fact.

Putnam also claims that predicative control is fundamental for Natural Sciences and makes objectivity possible. Cordero thinks that besides predicative-objectivity, so appreciated in Natural Sciences, there are other forms of objectivity. They might be forms grounded on other virtues than those mentioned by Putnain, e.g. the virtue of searching for human liberation. 